\title{
Changes of circulating neuregulin 4 and its relationship with 25-hydroxy vitamin $D$ and other diabetic vascular complications in patients with diabetic peripheral neuropathy
}

\author{
Pijun Yan ${ }^{1}{ }^{1}$, Zhihong Zhang ${ }^{2}$, Ying Miao ${ }^{1}$, Yong Xu' ${ }^{1}$, Jianhua Zhu ${ }^{1}$ and Qin Wan ${ }^{1 *}$
}

\begin{abstract}
Background: Neuregulin 4 (Nrg4) is a novel neurotrophic adipokine associated with the development of diabetic peripheral neuropathy (DPN), however, the pathological mechanism remains poorly understood. The purpose of our study was to investigate the association of circulating Nrg4 with DPN and 25-hydroxy vitamin D [25(OH)D], a multifunctional secosteroid hormone that regulates other neurotrophic factors and adipokines gene expression, and other diabetic vascular complications.
\end{abstract}

Methods: Circulating Nrg4 levels were measured with an ELISA kit in 164 newly diagnosed type 2 diabetes mellitus (nT2DM) patients. The relationship between circulating Nrg4 and DPN and other parameters was analyzed.

Results: Circulating Nrg4 levels were significantly lower in nT2DM patients with DPN than those without, and subjects in the highest quartile of circulating Nrg4 had significantly lower vibration perception threshold (VPT), the prevalence of DPN, the proportion of persons with VPT $>25 \mathrm{~V}$, and significantly higher circulating $25(\mathrm{OH}) \mathrm{D}$ (all $P<0.01$ ). Moreover, circulating Nrg4 was positively and independently associated with $25(\mathrm{OH}) \mathrm{D}$, and was negatively with VPT $(P<0.01$ or $P<0.05)$, but showed no associations with the prevalence of peripheral arterial disease, diabetic nephropathy, and diabetic retinopathy (all $P>0.05$ ). Additionally,the prevalence of DPN and risk of DPN development were progressively decreased with increasing circulating Nrg4 quartiles, independently of potential confounding factors.

Conclusions: These data demonstrate that decreased levels of circulating Nrg4 might lead to the development of DPN through its close interaction with circulating $25(\mathrm{OH}) \mathrm{D}$ not with other diabetic vascular complications. Further prospective studies are needed to identify our findings in these populations.

Keywords: Neuregulin 4, Diabetic peripheral neuropathy, Diabetic vascular, Complications, 25-hydroxy vitamin D

\section{Background}

Diabetic peripheral neuropathy (DPN), one of the most common diabetic microvascular complications, is characterized by numbness, pain, weakness, and loss of sensation in your hands, feet, or legs associated with

\footnotetext{
*Correspondence: wanqin231@126.com

${ }^{1}$ Department of Endocrinology, The Affiliated Hospital of Southwest Medical University, Luzhou, Sichuan 646000, China

Full list of author information is available at the end of the article
}

peripheral nerve dysfunction. DPN develops quite early during the disease, reaching at least $10-15 \%$ and possibly affecting around $50 \%$ of all diabetes patients as diabetes duration increases $[1,2]$. DPN is a major risk factor for foot ulcers and amputations, resulting in depression, lower life quality, limited mobility, social dysfunction, potential morbidity and mortality, and a huge economic burden [2,3]. However, to date, there has been no specific prevention and treatment for DPN other than strict glycemic control and symptomatic

c) The Author(s) 2020. This article is licensed under a Creative Commons Attribution 4.0 International License, which permits use, sharing, adaptation, distribution and reproduction in any medium or format, as long as you give appropriate credit to the original author(s) and the source, provide a link to the Creative Commons licence, and indicate if changes were made. The images or other third party material in this article are included in the article's Creative Commons licence, unless indicated otherwise in a credit line to the material. If material is not included in the article's Creative Commons licence and your intended use is not permitted by statutory regulation or exceeds the permitted use, you will need to obtain permission directly from the copyright holder. To view a copy of this licence, visit http://creativeco mmons.org/licenses/by/4.0/. The Creative Commons Public Domain Dedication waiver (http://creativecommons.org/publicdomain/ zero/1.0/) applies to the data made available in this article, unless otherwise stated in a credit line to the data. 
relief. Therefore, there is an urgent need to early detect and appropriately control novel modifiable risk factors associated with the development of DPN.

Neuregulin-4 (Nrg4) is an emerging multifunctional adipokine that is secreted predominantly by brown adipose tissue (BAT). Nrg4 acts via paracrine, autocrine or endocrine mechanisms by releasing the epidermal growth factor (EGF)-like domain after photolytic cleavage $[3,4]$. Numerous studies showed that decreased $\mathrm{Nrg} 4$ levels were closely associated with obesity, insulin resistance, diabetes mellitus, dyslipidemia, metabolic syndrome, non-alcoholic fatty liver disease, inflammation, oxidative stress, and macrovascular disease such as coronary artery disease, myocardial infarcts, subclinical cardiovascular disease (CVD) in rodents and humans [3, 5-15], while all above factors have been reported to contribute to the development of DNP $[1-3,16,17]$, suggesting indirectly that Nrg4 appears to play a crucial role in the development of DNP.

In accordance with the this hypothesis, it has been reported that Nrg4, a member of neuregulins, may have neuroprotective and neurotrophic effects and can promote neurite outgrowth and the development of neuronal progenitor stem cells, and modulate the growth and elaboration of pyramidal neuron dendrites, and spine formation in the striatum [18-21]. Recently, there is preliminary in vivo evidence that circulating $\mathrm{Nrg} 4$ levels were significantly decreased in newly diagnosed type 2 diabetes mellitus (nT2DM) patients with DPN [3], however, the pathological mechanism underlying the relationship between decreased plasma Nrg4 levels and increased risk of DPN remains poorly understood.

Vitamin D is a multifunctional secosteroid hormone that has a key role in bone homeostasis, and its status is the most commonly determined by measuring circulating 25-hydroxyvitamin $\mathrm{D}[25(\mathrm{OH}) \mathrm{D}]$. There are bodies of evidence showing that adequate status of vitamin D can modulate insulin sensitivity, immune and inflammatory responses, improve endothelial dysfunction and cardiometabolic health parameters [22-24], and vitamin D deficiency was associated with diabetes and its microand macrovascular complications such as DPN, diabetic nephropathy $(\mathrm{DN})$, diabetic retinopathy (DR), peripheral arterial disease (PAD), cerebral infarction, and cardiovascular disease and risk factors, such as hypertension, dyslipidemia, and obesity [25-28]. Previous evidence has demonstrated that $25(\mathrm{OH}) \mathrm{D}$ can up-regulate other neurotrophic factors, such as neurotrophin-3 (NT-3), nerve growth factor (NGF), and glial cell line-derived neurotrophic factor (GDNF) [29]. Moreover, it has been also reported that adipose tissue is the major site of vitamin D storage. Recent studies have demonstrated that vitamin D receptor (VDR) and vitamin D metabolizing enzymes are expressed in adipocytes [22].

Furthermore, it has been shown that vitamin D regulates adipocyte apoptosis as well as adipokines gene expression such as adiponectin and omentin-1 [22, 30]. Of note, Nrg4 as a good adipocytokine has similar features and functions to adiponectin and omentin-1, and also as a neurotrophic factor exerts neurotrophic and neuroprotective effects [3]. We hypothesized that circulating $25(\mathrm{OH}) \mathrm{D}$ is inversely associated with plasma Nrg4, and circulating $25(\mathrm{OH}) \mathrm{D}$ may partially mediate the association of plasma Nrg4 with the development of DPN.

Therefore, the study was conducted to investigate the relationship between plasma Nrg4 and DPN in a crosssectional population consisted of 164 Chinese patients with nT2DM. Moreover, we evaluated the potential associations among plasma Nrg4 and circulating 25(OH)D, and other diabetic micro- and macrovascular complications (DR, DN, and PAD).

\section{Methods \\ Study population}

164 patients with nT2DM between 44 and 87 years of age, from among inpatients in our department of endocrinology between January 2016 and January 2017, were recruited in the cross-sectional study. T2DM is diagnosed by an oral glucose tolerance test (OGTT) based on American Diabetes Association criteria. In the present study, nT2DM patients were split into two groups: 76 patients with DPN (DPN group) and 88 patients without DPN (no DPN group). All nT2DM patients didn't receive any lipid-lowering agents and anti-diabetic therapy, including diet control, exercise therapy, hypoglycemic agents and insulin. Excluded were patients with other endocrine disorders such as acromegaly, osteoporosis, thyroid disease, and parathyroid disease, type 1 diabetes, acute complications of diabetes, foot ulcers, limb amputations, cardiovascular and cerebrovascular disease, hypertension, nonalcoholic fatty liver disease, hepatic and renal failure, inflammatory or autoimmune diseases, active infection, cancer, pregnancy or lactation, current treatment with immunosuppressive drugs, systemic corticosteroids, prior history of knee or back surgery, any other etiological cause of peripheral neuropathy (e.g., toxin exposure, hereditary, carpal tunnel syndrome, peripheral polyneuritis, infectious polyneuritis, and vasculitis, cervical spondylosis, and lumbar spondylosis), cigarette smoking, alcohol consumption, and use of any drugs known to interfere with oxidant/antioxidant system and peripheral nerve function.

The study was performed in accordance with the ethical guidelines of the 1975 Declaration of Helsinki. The human research ethics committee of the Affiliated 
Hospital of Southwest Medical University approved the study, and informed consent was obtained from all nT2DM patients before any study-related procedure.

\section{Anthropometric and biochemical measurements}

Information concerning age, gender, medical history and lifestyle factors (smoking and alcohol consumption) was assessed by a standardized questionnaire. The height, body weight, and blood pressures were measured, as described previously [3]. Based on height and body weight measured, body mass index (BMI) of all patients was calculated.

Blood samples of study subjects were collected in the morning after an overnight fast or $2 \mathrm{~h}$ after OGTT. Plasma was obtained by centrifugation at $3500 \mathrm{rpm}$ for $10 \mathrm{~min}$ at $4{ }^{\circ} \mathrm{C}$ and subsequently stored at $-80{ }^{\circ} \mathrm{C}$ until analytical processing. Fasting blood glucose (FBG) and postprandial $2 \mathrm{~h}$ blood glucose (PBG) were measured by the glucose-oxidase method, and an anion exchange high performance liquid chromatography method was performed to determine levels of glycated hemoglobinA1C (HbA1c) (arkray ELUENT 80A, Japan). Lipid profiles, including total cholesterol (TC), triglyceride (TG), high density lipoprotein cholesterol (HDL-C), low-density lipoprotein cholesterol (LDL-C), and serum creatinine (Scr) were analyzed enzymatically using a 7060 full-automatic biochemical analyzer (Hitachi, Tokyo, Japan). Fasting insulin (Fins) level in plasma was determined using an electrochemiluminescence immunoassay (Roche Elecsys Insulin Test, Roche Diagnostics, Mannheim, Germany). The homeostasis model assessment of insulin resistance (HOMA-IR) was calculated to assess insulin resistance [3]. An electrochemiluminescence immunoassay was used to determine circulating concentration of 25(OH)D. Estimated glomerular filtration rate (eGFR) was calculated using Chronic Kidney Disease Epidemiology Collaboration (CKD-EPI) equa-tions modified by a Japanese coefficient [31]. Urinary microalbumin was measured in a random urine sample using an immunoturbidimetric test, and urinary creati-nine was measured enzymatically. The urine albumin-to-creatinine ratio (UACR) is expressed as milligram of albumin excreted pergram of urinary creatinine. DN was defined as the absence of signs or symptoms of other primary causes of kidney damage, the presence of albuminuria (UACR $\geq 30 \mathrm{mg} / \mathrm{g}$ creatinine) or an eGFR $<60 \mathrm{~mL} / \mathrm{min} / 1.73 \mathrm{~m}^{2}$ [31].

\section{Measurements of circulating Nrg4}

Circulating Nrg4 concentrations were also measured with an ELISA (Aviscera Biosciences, Santa Clara, CA). The detection sensitivity was $0.125-0.25 \mathrm{ng} / \mathrm{mL}$, and the standard linear range was $0.25-16.0 \mathrm{ng} / \mathrm{mL}$. Both intraand inter-assay CVs were smaller than $10 \%$.

\section{Foot examination and definition of DR}

All nT2DM patients were asked whether they had a history for claudication, and underwent a comprehensive foot examination including assessment of the pedal pulses. Then a diagnostic ankle-brachial index (ABI), measured by a continuous-wave Doppler ultrasound probe (Vista AVS, Summit Co., USA), was performed in nT2DM patients. Patients were diagnosed as having PAD, categorized as one of macrovascular complications, if an ABI value $<0.9$ on either limb [32]. All nT2DM patients were asked whether they had symptoms including pain, unpleasant abnormal sensations (sensory disturbance of glove and stocking type distribution, tingling, and burning), and numbness in patients' feet, legs and upper-limb, and undergone physical examination including ankle and knee reflexes examination. Then, quantitative sensory testing (QST), including assessment of pinprick sensation using a $40 \mathrm{~g}$ needle, vibrating perception threshold (VPT) using a neurothesiometer, and light touch perception using a 10-g monofilament, was performed to detect peripheral neuropathy in nT2DM subjects. VPT value of either limb exceeded $25 \mathrm{~V}$ (volt) was considered abnormal [3]. Patients were diagnosed as having DPN, as described previously [3].

A Canon CR-2 Digital Retinal Camera was performed to obtain two-field fundus photography of patient's eyes (Canon Inc., Kanagawa, Japan). The presence of DR was assessed by high-quality fundus photographs and an ophthalmologist who is knowledgeable and experienced in the management and treatment of DR.

\section{Statistical analysis}

All data was analyzed by the SPSS version 20.0 (Chicago, IL, USA). The normal distribution of all data was conducted with the Kolmogorov-Smirnov test, and the Levene homogeneity of variance test was performed to test homogeneity of variance.

Categorical variables are presented as numbers (percentages), otherwise, mean \pm standard deviation (SD) was given for continuous variables. Categorical variables between two groups were compared by $x^{2}$ test. Comparison between two groups was performed with Student's $t$ test for normally distributed continuous variables or Mann-Whitney U test for nonparametric distributed continuous variables. The subjects were divided into four groups based on circulating Nrg4 quartiles $(\mathrm{Q} 1<1.61 \mathrm{ng} / \mathrm{mL}, 1.61 \mathrm{ng} / \mathrm{mL} \leq \mathrm{Q} 2<2.71 \mathrm{ng} /$ $\mathrm{mL}, 2.71 \leq \mathrm{Q} 3 \leq 3.91 \mathrm{ng} / \mathrm{mL}, \mathrm{Q} 4>3.91 \mathrm{ng} / \mathrm{mL})$. Statistical difference was assessed using one-way analysis of variance (ANOVA) (continuous variables with 
normally distribution and homogeneity of variance), or the Kruskal-Wallis test (covariates with nonparametric distribution and/or variance uneven). The association between circulating Nrg4 and other variables was investigated by Pearson or Spearman bivariate correlation analysis; subsequently correlations between circulating Nrg4 levels and other variables were further determined with the partial correlation analysis, adjusting for age, gender, and BMI. Then, the independent variables associated with circulating Nrg4 in nT2DM patients were identified with multiple stepwise linear regression analysis. Model covariates included sex, age, BMI, SBP, DBP, FBG, PBG, HbA1C, FIns, HOMA-IR, TG, TC, HDL-C, LDL-C, UACR, eGFR, Scr, 25(OH) $\mathrm{D}, \mathrm{ABI}$, and VPT. All covariates were entered into the model at the same time as there was no evidence of collinearity based on individual variance inflation factor $(\mathrm{VIF})<10$, and tolerance statistics $>0.1$. Last, multivariable logistic regression analysis was also used to identify the association of circulating levels of Nrg4 with risk of DPN. Odds ratios (OR) and 95\% confidence intervals $(\mathrm{CI})$ were estimated. In all statistical tests, a $P$ value of $<0.05$ was considered to be statistically significant (two sided).

For sample size calculation, we used the following formula:

$$
\mathbf{N}=\frac{\left(\boldsymbol{q}_{1}^{-1}+\boldsymbol{q}_{2}^{-1}\right)\left(\boldsymbol{t}_{\boldsymbol{\alpha} / 2}+\boldsymbol{t}_{\boldsymbol{\beta}}\right)^{2} \boldsymbol{S}^{2}}{\boldsymbol{\delta}^{2}}
$$

where $\mathrm{N}$ is the total sample size (the sum of the sizes of both comparison groups). In our study, it is assumed that test level $\alpha=0.05, \beta=0.1$, and the population mean difference between nT2DM patients without DPN group and nT2DM patients with DPN group is $1.5(\delta=1.5)$. Also, we assume that the sample size ratio between nT2DM patients without DPN group and nT2DM patients with DPN group is 1:1.5. Accordingly, q1 $($ nT2DM patients with DPN group $)=1.5 /(1+1.5)=0.6$, and q2 (nT2DM patients without DPN group) $=1 /$ $(1+1.5)=0.4$. Previous work has proposed that the SD of Nrg4 for nT2DM patients without DPN group is 1.5 $(\mathrm{S}=1.5)$ [3]. Then, all parameters are included in the above formula, and we get the following result:

$$
\begin{aligned}
N & =\frac{\left[(0.6)^{-1}+(0.4)^{-1}\right](1.960+1.282)^{2} \times 2^{2}}{1.5^{2}} \\
& =77.85=78
\end{aligned}
$$

From the result, we known that the minimum sample size in nT2DM patients without DPN group is 31 , and the minimum sample size in nT2DM patients with DPN group is 47 . In other words, this study have test efficacy of 0.90 at a two-sided $\alpha$ of 0.05 as long as the
Table 1 Circulating Nrg4 levels and other clinical

\begin{tabular}{|c|c|c|c|}
\hline Variables & $\begin{array}{l}\text { No DPN } \\
(n=88)\end{array}$ & $\begin{array}{l}\text { DPN } \\
(n=76)\end{array}$ & $P$ \\
\hline Men/women & $32 / 56$ & $46 / 30$ & 0.002 \\
\hline Age (years) & $63.90 \pm 8.44$ & $63.88 \pm 9.93$ & 0.991 \\
\hline BMI $\left(\mathrm{kg} / \mathrm{m}^{2}\right)$ & $25.02 \pm 3.96$ & $26.10 \pm 3.47$ & 0.067 \\
\hline $\mathrm{SBP}(\mathrm{mmHg})$ & $123.45 \pm 10.76$ & $125.66 \pm 12.78$ & 0.232 \\
\hline $\mathrm{DBP}(\mathrm{mmHg})$ & $73.68 \pm 8.48$ & $74.05 \pm 9.84$ & 0.796 \\
\hline $\mathrm{FBG}(\mathrm{mmol} / \mathrm{L})$ & $10.71 \pm 3.30$ & $11.38 \pm 3.51$ & 0.212 \\
\hline PBG (mmol/L) & $18.51 \pm 6.34$ & $19.42 \pm 5.43$ & 0.329 \\
\hline $\mathrm{HbA1c}(\mathrm{mmol} / \mathrm{mol})$ & $86.84 \pm 26.159$ & $85.56 \pm 21.95$ & 0.736 \\
\hline $\mathrm{HbA1c}(\%)$ & $10.10 \pm 2.39$ & $9.98 \pm 2.01$ & 0.736 \\
\hline FIns $(\mu \mathrm{U} / \mathrm{ml})$ & $11.23 \pm 6.52$ & $10.03 \pm 5.60$ & 0.183 \\
\hline HOMA-IR & $5.20 \pm 3.53$ & $4.97 \pm 3.03$ & 0.654 \\
\hline $\mathrm{TC}(\mathrm{mmol} / \mathrm{L})$ & $4.27 \pm 1.23$ & $4.62 \pm 1.32$ & 0.193 \\
\hline $\mathrm{TG}(\mathrm{mmol} / \mathrm{L})$ & $2.12 \pm 1.91$ & $2.17 \pm 2.02$ & 0.403 \\
\hline $\mathrm{HDL}-\mathrm{C}(\mathrm{mmol} / \mathrm{L})$ & $1.12 \pm 0.31$ & $1.13 \pm 0.42$ & 0.970 \\
\hline LDL-C (mmol/L) & $2.53 \pm 1.00$ & $2.80 \pm 0.95$ & 0.086 \\
\hline UACR (mg/g) & $42.43 \pm 49.29$ & $41.71 \pm 56.23$ & 0.377 \\
\hline $\operatorname{Scr}(\mu \mathrm{mol} / \mathrm{L})$ & $68.93 \pm 18.43$ & $70.39 \pm 19.53$ & 0.622 \\
\hline $\operatorname{eGFR}\left(\mathrm{mL} / \mathrm{min} / 1.73 \mathrm{~m}^{2}\right)$ & $87.06 \pm 17.89$ & $89.76 \pm 18.23$ & 0.342 \\
\hline $\mathrm{VPT}(\mathrm{V})$ & $14.19 \pm 5.47$ & $24.16 \pm 11.55$ & 0.000 \\
\hline $\mathrm{ABI}$ & $1.03 \pm 0.12$ & $1.03 \pm 0.15$ & 0.581 \\
\hline $25(\mathrm{OH}) \mathrm{D}(\mathrm{ng} / \mathrm{mL})$ & $17.36 \pm 5.57$ & $14.51 \pm 3.99$ & 0.000 \\
\hline $\operatorname{Nrg} 4(\mathrm{ng} / \mathrm{mL})$ & $3.32 \pm 1.62$ & $2.49 \pm 1.49$ & 0.001 \\
\hline $\operatorname{PAD}(n, \%)$ & $6(6.81)$ & $6(7.89)$ & 0.792 \\
\hline DN (n, \%) & $37(42.05)$ & $29(38.16)$ & 0.613 \\
\hline DR (n, \%) & $10(11.36)$ & $10(13.16)$ & 0.726 \\
\hline
\end{tabular}
characteristics between $\mathrm{nt} 2 \mathrm{dm}$ patients with and without $\operatorname{DPN}(\bar{x} \pm s)$

Data are mean \pm SD

$S D$ standard deviation, $B M I$ body mass index, $S B P$ systolic blood pressure, $D B P$ diastolic blood pressure, $F B G$ fasting blood glucose, $P B G$ postprandial $2 \mathrm{~h}$ blood glucose, HbA1c glycated hemoglobin A1c, Flns fasting plasma insulin, HOMA-IR homeostasis model assessment of insulin resistance, $T C$ total cholesterol, TG triglyceride, $H D L-C$ high-density lipoprotein cholesterol, $L D L-C$ low-density lipoprotein cholesterol, UACR urine albumin-to-creatinine ratio, $S c r$ serum creatinine, 25(OH)D 25-hydroxyvitamin D, Nrg4 Neuregulin 4, eGFR estimated glomerular filtration rate, VPT vibration perceptionthreshold,DNdiabetic nephropathy, $P A D$ peripheral arterial disease, $D R$ diabetic retinopathy, $D P N$ diabetic peripheral neuropathy

sample size in nT2DM patients without DPN group is $\geq 31$ and the sample size in nT2DM patients with DPN group is $\geq 47$.

\section{Results}

\section{Circulating Nrg4 levels and other clinical characteristics of studied population}

The anthropometric, biochemical and clinical parameters of studied population are shown in Table 1. BMI, age, lipid profiles, blood pressure, plasma glucose, insulin, Scr, eGFR, UACR, ABI, and the prevalence of DN, $\mathrm{DR}$ and PAD were similar between the two groups (all 
$P>0.05)$. When compared with nT2DM patients with no DPN, those with DPN had significantly more men, higher VPT values, and lower levels of circulating Nrg4 and $25(\mathrm{OH}) \mathrm{D}(P<0.01$ or $P<0.05)$.

\section{Clinical and biochemical characteristics across quartiles of circulating Nrg4 in all nT2DM patients}

Table 2 presents the clinical and biochemical characteristics across quartiles of circulating Nrg4 levels. Circulating levels of $\mathrm{Nrg} 4$ and 25(OH)D, VPT values, age, prevalence of DPN, the proportion of individuals with VPT $>25 \mathrm{~V}$ and three or more abnormal DPN screening were significantly different across quartiles of plasma Nrg4 $(P<0.01$ or $P<0.05)$. Compared to subjects in the lowest quartile of circulating Nrg4, those in the highest quartile had significantly lower VPT values, the prevalence of DPN, the proportion of persons with VPT $>25 \mathrm{~V}$ and three or more abnormal DPN screening, and significantly higher levels of circulating $\mathrm{Nrg} 4$ and $25(\mathrm{OH}) \mathrm{D}$, and older age $(P<0.01$ or $P<0.05)$. However, no significant differences among the four quartile groups were observed for UACR, eGFR, ABI, and the prevalence of DN, DR and PAD (all $P>0.05)$.

\section{Association of circulating Nrg4 with 25(OH)D, diabetic vascular complications, and other parameters in study subjects}

Next, we analyzed the relationships of circulating Nrg4 levels with 25(OH)D, diabetic vascular complications, other parameters by using simple correlations.

Table 2 clinical and biochemical characteristics by quartiles of circulating Nrg4 in all nT2DM patients

\begin{tabular}{|c|c|c|c|c|c|}
\hline Covariates & Quartile 1 & Quartile 2 & Quartile 3 & Quartile 4 & $\mathbf{P}$ \\
\hline Sample size & 41 & 41 & 41 & 41 & \\
\hline Nrg4 (ng/ml) & $1.18 \pm 0.31$ & $2.14 \pm 0.31^{* *}$ & $3.26 \pm 0.33^{* * \# \#}$ & $5.18 \pm 1.08^{* * \# \# \$}$ & 0.000 \\
\hline Men/women & $24 / 17$ & $20 / 21$ & $15 / 26$ & $19 / 22$ & 0.263 \\
\hline Age (years) & $65.46 \pm 9.70$ & $61.29 \pm 7.96^{*}$ & $62.37 \pm 8.92$ & $66.44 \pm 9.19^{\$}$ & 0.030 \\
\hline $\mathrm{BMI}\left(\mathrm{kg} / \mathrm{m}^{2}\right)$ & $26.55 \pm 3.69$ & $25.79 \pm 3.95^{*}$ & $25.02 \pm 3.90$ & $24.72 \pm 3.39^{*}$ & 0.119 \\
\hline $\mathrm{SBP}(\mathrm{mmHg})$ & $123.51 \pm 11.16$ & $122.56 \pm 11.50$ & $124.83 \pm 13.19$ & $127.00 \pm 10.98$ & 0.351 \\
\hline $\mathrm{DBP}(\mathrm{mmHg})$ & $72.07 \pm 11.29$ & $73.37 \pm 9.32$ & $75.73 \pm 8.18$ & $74.24 \pm 7.05$ & 0.333 \\
\hline $\mathrm{FBG}(\mathrm{mmol} / \mathrm{L})$ & $11.48 \pm 3.73$ & $11.79 \pm 3.69$ & $10.38 \pm 2.83$ & $10.44 \pm 3.18$ & 0.140 \\
\hline PBG (mmol/L) & $18.82 \pm 4.76$ & $19.19 \pm 5.92$ & $18.91 \pm 7.62$ & $18.82 \pm 5.26$ & 0.774 \\
\hline $\mathrm{HbA1c}(\mathrm{mmol} / \mathrm{mol})$ & $84.98 \pm 23.16$ & $87.45 \pm 20.99$ & $85.63 \pm 25.90$ & $86.93 \pm 27.21$ & 0.966 \\
\hline $\mathrm{HbA1c}(\%)$ & $9.93 \pm 2.12$ & $10.15 \pm 1.92$ & $9.99 \pm 2.37$ & $10.10 \pm 2.49$ & 0.966 \\
\hline Flns ( $\mu \mathrm{U} / \mathrm{ml})$ & $10.05 \pm 6.35$ & $11.71 \pm 6.29$ & $10.26 \pm 5.32$ & $10.68 \pm 6.56$ & 0.624 \\
\hline HOMA-IR & $4.76 \pm 2.96$ & $6.12 \pm 3.85$ & $4.65 \pm 2.75$ & $4.84 \pm 3.44$ & 0.145 \\
\hline $\mathrm{TC}(\mathrm{mmol} / \mathrm{L})$ & $4.42 \pm 1.23$ & $4.37 \pm 1.33$ & $4.36 \pm 1.18$ & $4.56 \pm 1.40$ & 0.893 \\
\hline $\mathrm{TG}(\mathrm{mmol} / \mathrm{L})$ & $2.30 \pm 2.69$ & $2.32 \pm 1.53$ & $1.55 \pm 0.80$ & $2.42 \pm 2.21^{*}$ & 0.082 \\
\hline $\mathrm{HDL}-\mathrm{C}(\mathrm{mmol} / \mathrm{L})$ & $1.07 \pm 0.31$ & $1.10 \pm 0.46$ & $1.16 \pm 0.32^{*}$ & $1.16 \pm 0.36^{* *}$ & 0.543 \\
\hline LDL-C (mmol/L) & $2.67 \pm 0.91$ & $2.51 \pm 0.92$ & $2.74 \pm 1.03$ & $2.70 \pm 1.08$ & 0.731 \\
\hline UACR (mg/g) & $39.46 \pm 8.70$ & $38.02 \pm 6.49$ & $38.07 \pm 7.79$ & $52.84 \pm 9.54$ & 0.123 \\
\hline $\mathrm{Scr}(\mu \mathrm{mol} / \mathrm{L})$ & $70.56 \pm 21.03$ & $69.83 \pm 18.44$ & $65.60 \pm 16.44$ & $72.42 \pm 19.46$ & 0.417 \\
\hline eGFR (mL/min/1.73 m²) & $88.76 \pm 22.44$ & $89.84 \pm 15.55$ & $90.76 \pm 16.95$ & $83.89 \pm 16.29$ & 0.319 \\
\hline $\mathrm{ABI}$ & $1.04 \pm 0.07$ & $1.05 \pm 0.11$ & $1.02 \pm 0.15$ & $1.01 \pm 0.18$ & 0.880 \\
\hline $\operatorname{VPT}(\mathrm{V})$ & $27.15 \pm 11.37$ & $18.80 \pm 10.06^{* *}$ & $14.29 \pm 5.77^{* *}$ & $15.00 \pm 6.78^{* *}$ & 0.000 \\
\hline $25(\mathrm{OH}) \mathrm{D}(\mathrm{ng} / \mathrm{mL})$ & $15.91 \pm 5.35$ & $14.11 \pm 3.05^{* *}$ & $15.76 \pm 4.51$ & $18.37 \pm 6.15^{\# \#}$ & 0.007 \\
\hline DPN, n (\%) & $28(68.29)$ & $19(46.34)$ & $16(39.02)^{*}$ & $13(31.71)^{* *}$ & 0.006 \\
\hline VPT $>25 \mathrm{~V}, \mathrm{n}(\%)$ & $28(68.29)$ & $10(24.39)^{* *}$ & $3\left(7.32^{* * \# \#}\right.$ & $2\left(4.88^{* * \# \$ \$}\right.$ & 0.000 \\
\hline Abnormal pricking sensation, n (\%) & $34(82.93)$ & $22(53.66)$ & $25(60.98)$ & $22(53.66)$ & 0.250 \\
\hline Abnormal nylon monofilament, n (\%) & $8(19.51)$ & $12(29.27)$ & $11(26.83)$ & $11(26.83)$ & 0.808 \\
\hline Reflex abnormalities, n (\%) & $6(14.63)$ & $5(12.20)$ & $3(7.32)$ & $2(4.88)$ & 0.431 \\
\hline Two abnormal DPN screening, n (\%) & $20(48.78)$ & $16(39.02)$ & $15(36.59)$ & $12(29.27)$ & 0.340 \\
\hline Three or more abnormal DPN screening, n (\%) & $8(19.51)$ & $3(7.32)$ & $1(2.44)^{* *}$ & $1(2.44)^{* *}$ & 0.012 \\
\hline DN, n (\%) & $16(39.02)$ & $18(43.90)$ & $13(31.71)$ & $19(46.34)$ & 0.549 \\
\hline $\mathrm{DR}, \mathrm{n}(\%)$ & $4(9.76)$ & $6(14.63)$ & $4(9.76)$ & $6(14.63)$ & 0.824 \\
\hline PAD, n (\%) & $3(7.32)$ & $1(2.44)$ & $4(9.76)$ & $4(9.76)$ & 0.543 \\
\hline
\end{tabular}

${ }^{*} P<0.05,{ }^{* *} P<0.01$ compared with quartile 1 group; ${ }^{\#}<0.05,{ }^{\# \#} 0.01$ compared with quartile 2 group; ${ }^{\$}<0.05,{ }^{\$ \$} 0.01$ compared with quartile 3 group 
The simple correlation analysis demonstrated that circulating Nrg4 levels were positively associated with HDL-C and $25(\mathrm{OH}) \mathrm{D}$, and negatively with BMI, VPT values, the prevalence of DPN, and the proportion of individuals with VPT $>25 \mathrm{~V}(P<0.01$ or $P<0.05$; Table 3$)$. Also, after adjustment for sex, age, and $\mathrm{BMI}$, this positive association of circulating Nrg4 with of $25(\mathrm{OH}) \mathrm{D}(P<0.01)$, and this negative associations between circulating Nrg4 and VPT values, the proportion of individuals with $\mathrm{VPT}>25 \mathrm{~V}$, and the prevalence of DPN remained statistically significant (all $P<0.01$; Table 3). Then, multiple stepwise regressions analysis was used to determine the main determinants of circulating levels of Nrg4. We showed that circulating levels of $25(\mathrm{OH}) \mathrm{D}$, VPT values, SBP, and LDL-C were dependent related factors with circulating Nrg4 (Table 3). The multiple regression equation was:

$$
\begin{aligned}
\mathrm{Y}_{\mathrm{Nrg} 4}= & -0.274-0.059 X_{\mathrm{VPT}}+0.070 X_{25(\mathrm{OH}) \mathrm{D}} \\
& +0.021 \mathrm{X}_{\mathrm{SBP}}+0.245 X_{\mathrm{LDL}-\mathrm{C}} \\
& \left(\mathrm{R}=0.476, \mathrm{R}^{2}=0.207\right)
\end{aligned}
$$

\section{Association between quartiles of circulating Nrg4 and $25(\mathrm{OH}) \mathrm{D}$ and risk of DPN in study subjects}

To assess whether higher circulating Nrg4 can decrease the risk of development of DPN, multivariate logistic regression analysis was mapped. All subjects were categorized into four quartile groups according to circulating Nrg4. As shown in Table 4, the prevalence of DPN and risk of DPN development were progressively decreased with increasing circulating Nrg4 quartiles ( $P$ for trend $<0.01$ or $P$ for trend $P<0.05)$. When

\begin{tabular}{|c|c|c|c|c|c|c|c|}
\hline \multirow[t]{2}{*}{ Variable } & \multicolumn{4}{|l|}{ Simple } & \multicolumn{3}{|l|}{ Multiple } \\
\hline & $\mathbf{R}$ & P-value & Adjusted r & Adjusted P-value & $\beta$ & Standardized $\beta$ & P-value \\
\hline Sex & 0.125 & 0.110 & - & - & & & \\
\hline Age & 0.075 & 0.340 & - & - & & & \\
\hline BMI & -0.165 & 0.035 & - & - & & & \\
\hline SBP & 0.115 & 0.142 & 0.125 & 0.114 & 0.021 & 0.150 & 0.034 \\
\hline DBP & 0.083 & 0.290 & 0.104 & 0.190 & & & \\
\hline FBG & -0.123 & 0.116 & -0.077 & 0.329 & & & \\
\hline PBG & -0.032 & 0.680 & -0.017 & 0.834 & & & \\
\hline $\mathrm{HbA} 1 \mathrm{c}$ & 0.035 & 0.653 & 0.031 & 0.697 & & & \\
\hline FIns & 0.010 & 0.894 & 0.006 & 0.937 & & & \\
\hline HOMA-IR & -0.061 & 0.440 & -0.032 & 0.690 & & & \\
\hline $\mathrm{TC}$ & 0.110 & 0.159 & 0.105 & 0.184 & & & \\
\hline $\mathrm{TG}$ & -0.034 & 0.661 & 0.042 & 0.598 & & & \\
\hline HDL-C & 0.148 & 0.059 & 0.109 & 0.169 & & & \\
\hline LDL-C & 0.117 & 0.136 & 0.113 & 0.155 & 0.245 & 0.149 & 0.035 \\
\hline UACR & 0.122 & 0.120 & 0.103 & 0.19 & & & \\
\hline eGFR & -0.078 & 0.322 & -0.076 & 0.337 & & & \\
\hline Scr & -0.005 & 0.947 & 0.058 & 0.469 & & & \\
\hline $25(\mathrm{OH}) \mathrm{D}$ & 0.194 & 0.013 & 0.240 & 0.002 & 0.070 & 0.220 & 0.002 \\
\hline$A B \mid$ & -0.011 & 0.893 & 0.000 & 0.997 & & & \\
\hline VPT & -0.412 & 0.000 & -0.360 & 0.000 & -0.059 & -0.372 & 0.000 \\
\hline DPN & -0.276 & 0.000 & -0.218 & 0.005 & & & \\
\hline $\mathrm{VPT}>25 \mathrm{~V}$ & -0.506 & 0.000 & -0.433 & 0.000 & & & \\
\hline Abnormal prickingsensation & -0.078 & 0.322 & 0.130 & 0.100 & & & \\
\hline Abnormal nylon monofilament & 0.017 & 0.832 & -0.079 & 0.319 & & & \\
\hline Reflex abnormalities & -0.116 & 0.139 & -0.067 & 0.401 & & & \\
\hline DN & 0.000 & 0.999 & 0.045 & 0.571 & & & \\
\hline DR & 0.003 & 0.972 & 0.014 & 0.864 & & & \\
\hline PAD & 0.055 & 0.485 & 0.010 & 0.896 & & & \\
\hline
\end{tabular}

Table 3 Linear correlation and multiple regression analysis of variables associated with circulating Nrg4 in study subjects

In multiple linear stepwise regression analysis, values included for analysis in nT2DM patients were sex, age, BMI, SBP, DBP, FBG, PBG, HbA1C, FIns, HOMA-IR, TG, TC, HDL-C, LDL-C, UACR, eGFR, Scr, 25(OH)D, ABI, and VPT 
Table 4 Association between quartiles of circulating Nrg4 and 25(OH)D and risk of DPN in all nT2DM patients

\begin{tabular}{|c|c|c|c|c|c|}
\hline & Quartile 1 & Quartile 2 & Quartile 3 & Quartile 4 & $P$ for trend \\
\hline \multicolumn{6}{|l|}{ Circulating Nrg4 level } \\
\hline Circulating Nrg4 (ng/ml) & $1.18 \pm 0.31$ & $2.14 \pm 0.31$ & $3.26 \pm 0.33$ & $5.18 \pm 1.08$ & 0.000 \\
\hline Prevalence of DPN & $28(68.29)$ & $19(46.34)$ & $16(39.02)$ & $13(31.71)$ & 0.006 \\
\hline Model 1 & 1 & $0.401(0.163-0.986) 0.047$ & $0.297(0.120-0.738) 0.009$ & $0.216(0.085-0.546) 0.001$ & 0.008 \\
\hline Model 2 & 1 & $0.443(0.173-1.134) 0.089$ & $0.384(0.148-0.996) 0.049$ & $0.250(0.095-0.659) 0.005$ & 0.040 \\
\hline Model 3 & 1 & $0.336(0.102-1.104) 0.072$ & $0.282(0.082-0.966) 0.044$ & $0.115(0.032-0.417) 0.001$ & 0.026 \\
\hline Model 4 & 1 & $0.363(0.109-1.208) 0.099$ & $0.297(0.085-1.037) 0.057$ & $0.100(0.026-0.392) 0.001$ & 0.027 \\
\hline Model 5 & 1 & $0.304(0.086-1.071) 0.064$ & $0.213(0.053-0.865) 0.030$ & $0.116(0.027-0.494) 0.004$ & 0.047 \\
\hline \multicolumn{6}{|l|}{ Circulating 25(OH)D level } \\
\hline Circulating 25(OH)D (ng/ml) & $10.44 \pm 2.16$ & $14.25 \pm 0.61$ & $16.69 \pm 0.68$ & $22.77 \pm 4.22$ & 0.000 \\
\hline Prevalence of DPN & $21(51.22)$ & $22(53.66)$ & $23(56.10)$ & $10(24.39)$ & 0.013 \\
\hline Model 1 & 1 & $1.103(0.463-2.624) 0.825$ & $1.103(0.714-1.704) 0.658$ & $0.675(0.493-0.923) 0.014$ & 0.026 \\
\hline Model 2 & 1 & $1.139(0.472-2.745) 0.773$ & $1.091(0.693-1.716) 0.707$ & $0.681(0.493-0.940) 0.020$ & 0.032 \\
\hline Model 3 & 1 & $0.787(0.287-2.160) 0.642$ & $1.081(0.627-1.864) 0.779$ & $0.586(0.396-0.867) 0.007$ & 0.040 \\
\hline Model 4 & 1 & $0.662(0.221-1.982) 0.461$ & $1.060(0.604-1.860) 0.840$ & $0.564(0.367-0.868) 0.009$ & 0.028 \\
\hline Model 5 & 1 & $0.673(0.209-2.173) 0.508$ & $1.103(0.622-1.954) 0.738$ & $0.586(0.375-0.918) 0.020$ & 0.098 \\
\hline
\end{tabular}

Data are expressed as $\mathrm{OR}(95 \% \mathrm{Cl})+P$ value, unless stated otherwise

Model 1: crude model

Model 2: Model $1+$ sex, age, BMI

Model 3: Model 2+SBP, DBP, TC, TG, FBG, PBG, HbA1C, FIns, HOMA-IR

Model 4: Model 3+DR, DN, PAD

Model 5: Model 4+25(OH)D or Nrg4

$O R$ odds ratio; $\mathrm{Cl}$ confidence interval

compared with those in the lowest quartile, nT2DM patients in the highest circulating Nrg4 quartile had significantly lower risk of DPN development before and after adjustment for gender, age, BMI, blood pressure, lipid profiles, blood glucose, insulin, and the prevalence of DR, DN, and PAD ( $P$ for trend $<0.05$ ). This association between circulating Nrg4 and risk of DPN development remained significant after further adjustment for $25(\mathrm{OH}) \mathrm{D} \quad(\mathrm{OR}=0.116 ; 95 \%$ CI $0.027-0.494 ; P<0.05)$. Likewise, all subjects were categorized into four quartile groups according to circulating $25(\mathrm{OH}) \mathrm{D}$. The result suggest that the prevalence of DPN and risk of DPN development were progressively decreased with increasing circulating 25(OH)D quartiles $(P$ for trend $P<0.05)$. When compared with those in the lowest quartile, nT2DM patients in the highest circulating $25(\mathrm{OH})$ D quartile had significantly lower risk of DPN development before and after adjustment for gender, age, BMI, blood pressure, lipid profiles, blood glucose, insulin, and the prevalence of DR, DN, and PAD ( $P$ for trend $<0.05$ ). This association between circulating $25(\mathrm{OH}) \mathrm{D}$ and risk of DPN development tended to be statistically significant after further adjustment for Nrg4 $(\mathrm{OR}=0.586 ; 95 \% \mathrm{CI}$ $0.375-0.918 ; P=0.098$ ).
The predictive value of circulating $\mathrm{Nrg} 4$ and 25(OH)D in detecting DPN

To explore the predictive value of circulating $\mathrm{Nrg} 4$ and 25(OH)D for DPN, we analyzed the ROC curves of circulating Nrg4 and 25(OH)D. The results revealed that the best cutoff value for circulating Nrg4 to predict DPN was $3.03 \mathrm{ng} / \mathrm{ml}$ (sensitivity: $55.7 \%$, specificity: $71.1 \%$, and AUC 0.660), and the best cutoff value for circulating $25(\mathrm{OH}) \mathrm{D}$ to predict DPN was $16.491 \mathrm{ng} / \mathrm{ml}$ (sensitivity: $52.3 \%$, specificity: $76.3 \%$, and AUC 0.635 ) in patients with nT2DM (Fig. 1).

\section{Discussion}

To the best of our knowledge, this was the first study to illustrate the potential association of circulating Nrg4 with $25(\mathrm{OH}) \mathrm{D}$ and other diabetic vascular complications in Chinese patients with nT2DM, and again explore the role of circulating Nrg4 in the development of DPN. We found that circulating Nrg4 significantly decreased in nT2DM patients with DPN, and the risk of DPN was progressively decreased with increasing circulating Nrg4 quartiles even after adjustment for potential confounding factors. More importantly, our study showed for the first time that circulating $\mathrm{Nrg} 4$ was positively 


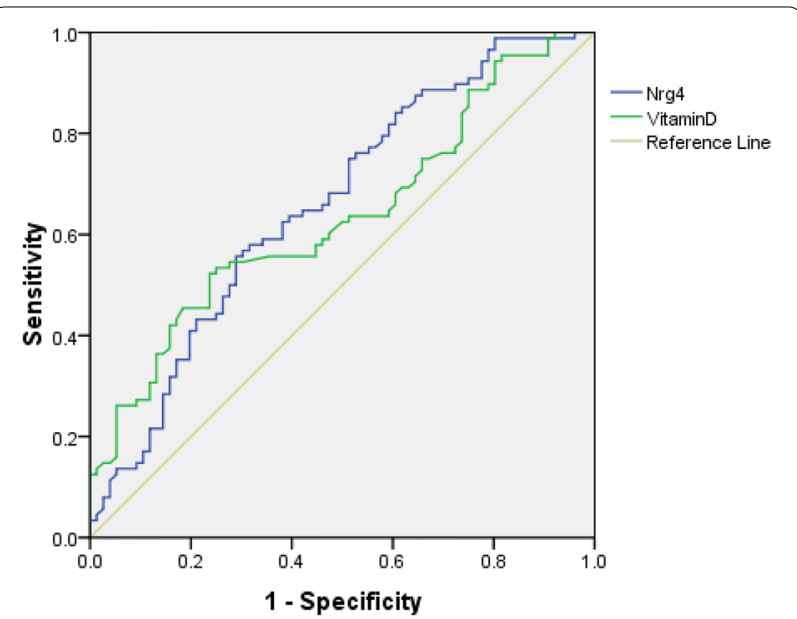

Fig. $1 \mathrm{ROC}$ analysis of circulating $\mathrm{Nrg} 4$ and $25(\mathrm{OH}) \mathrm{D}$ to indicate DPN for nT2DM patients. For circulating Nrg4, AUC $=0.660 ; 95 \%$ $\mathrm{Cl} 0.576-0.744 ; P=0.000$; identified Nrg4 cutoff value $=3.03 \mathrm{ng} /$ ml; Youden index $=0.268$; sensitivity: $55.7 \%$; specificity: $71.1 \%$; For circulating 25(OH)D, AUC $=0.635 ; 95 \% \mathrm{Cl} 0.551-0.720$; $P=0.003$; identified $25(\mathrm{OH}) \mathrm{D}$ cutoff value $=16.491 \mathrm{ng} / \mathrm{ml}$; Youden index $=0.286$; sensitivity: $52.3 \%$; specificity: $76.3 \%$

and independently related to $25(\mathrm{OH}) \mathrm{D}$ levels but not related to the prevalence of $\mathrm{DN}, \mathrm{DR}$, and PAD.

Nrg4 is a novel and predominantly BAT-secreted adipokine which was upregulated in white fat upon cold exposure [3]. Animal studies have shown that Nrg4 could prevent weight gain, attenuate diet-induced insulin resistance and hepatic steatosis through markedly mitigating hepatic lipogenic signaling, and maintain glycolipid balance [7]. Rosell et al. demonstrated that Nrg4-contained medium from PC12-HER4 cells can promote neurite outgrowth [20]. This was also observed in conditioned medium from transfected Cos7 cells or a chemically synthesized and refolded peptide [21]. Paramo and colleagues performed a study on the striatal Medium spiny neurons (MSN) of $\mathrm{Nrg}^{+/+}$and $\mathrm{Nrg} 4^{-/-}$mice in vivo and in vitro, and showed that $\mathrm{Nrg} 4$ was a prominent novel physiological regulator of the formation and maturation of dendritic spines and the growth and elaboration of MSN dendrites [33]. In humans, it has been reported that levels of adipose tissues Nrg4 mRNA and circulating Nrg4 were significantly reduced in T2DM patients compared with healthy individuals [6,34], and circulating Nrg4 was further markedly decreased in T2DM patients with DPN, and can predict DPN [3, 35]. Consistent with previous studies and our early report [3], we again confirmed that circulating Nrg4 significantly reduced in nT2DM patients with DPN, and was negatively and independently associated with VPT, a useful and reliable marker for early screening DPN and reflecting the clinical severity, and subjects in the highest circulating Nrg4 quartile had significantly lower VPT, the presence of DPN, the proportion of individuals with VPT $>25 \mathrm{~V}$, and three or more abnormal DPN screening as compared to those in the lowest quartile, indicating that decreased circulating Nrg4 may play a crucial role in the pathogenesis of DPN. Moreover, multivariate logistic regression analysis showed that the prevalence of DPN and risk of DPN development were progressively decreased with increasing circulating $\mathrm{Nrg} 4$ quartiles, independently of potential confounding factors, further implying a complex mechanistic association between decreased circulating Nrg4 and the development of DPN, however, the exact mechanism of pathogenesis remains unclear.

Several lines of evidence suggest that vitamin D can repair nociceptor function, elevate the pain threshold, modulate neurotransmission, contribute to synaptic plasticity, and has neurotrophic effects on nerve function and nerve growth factors [28]. Previous studies have demonstrated that vitamin D deficiency may play an important role in the development of DPN [26, 28, 36]. Our study provides further evidence that supports the potential role of vitamin D deficiency in the development of DPN, since we found that diabetic patients with DPN had significantly lower circulating $25(\mathrm{OH}) \mathrm{D}$ compared with those without, and the prevalence of DPN and risk of DPN development were progressively decreased with increasing circulating $25(\mathrm{OH}) \mathrm{D}$ quartiles. More importantly, circulating 25(OH)D was negatively and independently associated with DPN development, and can predict DPN. Interestingly, our study, for the first time, showed that circulating $25(\mathrm{OH}) \mathrm{D}$ correlated positively and independently with $\mathrm{Nrg} 4$, and circulating $25(\mathrm{OH}) \mathrm{D}$ increased along with increasing Nrg4 quartiles, suggesting that there seems to be a close association between circulating $\mathrm{Nrg} 4$ and 25(OH)D levels. Experimental studies suggest that vitamin $\mathrm{D}$ can up-regulate the synthesis of the neurotrophins such as NGF, GDNF, and regulate neuronal $\mathrm{Ca}^{2+}$ homeostasis and neuronal differentiation and maturation [28, 29, 37]. Conversely, low vitamin D levels have been linked to reduced levels of neurotrophins in animal models, with treatment via a vitamin $\mathrm{D}$ derivative shown to induce nerve growth factor [38]. Nrg4, member of the EGF family of extracellular ligands, exerts neurotrophic and neuroprotective effects as a neurotrophic factor [3]. Therefore, it is possible that circulating Nrg4 appears to be regulated by $25(\mathrm{OH}) \mathrm{D}$, and lower $25(\mathrm{OH}) \mathrm{D}$ may lead to reduced circulating Nrg4. Recent studies have demonstrated that VDR is expressed in adipocytes, and vitamin $D$ regulates adipokines gene expression such as adiponectin and omentin-1 $[22,30]$. Gupta et al. showed that a diet rich in VD increased adiponectin synthesis in swine epicardial adipose tissue [39]. Further, a direct effect of 1a, $25-(\mathrm{OH}) 2$ vitamin D3 on adipocytes was demonstrated, 
with adiponectin and its multimeric forms upregulated by 1a, 25- $(\mathrm{OH}) 2$ vitamin D3 treatment at low pharmacological concentrations [24]. Moreover, evidence suggests that, in humans, adiponectin synthesis and secretion is positively controlled by serum $25(\mathrm{OH}) \mathrm{D}$, and circulating $25(\mathrm{OH}) \mathrm{D}$ was positively correlated with serum adiponectin $[22,24,27,40]$. Regarding the effect of vitamin D on omentin-1, Dikker et al. reported that women with normal vitamin D levels had significantly higher serum omentin-1 levels, and circulating vitamin D was positively correlated with serum omentin-1 [30]. Of note, $\mathrm{Nrg} 4$ as a good adipocytokine has similar features and functions to adiponectin and omentin, and Nrg4 can promote mRNA expression of several beneficial adipokines, such as adiponectin, adipsin, and vascular endothelial growth factor $\alpha$ [41]. Hence, it is likely that $25(\mathrm{OH}) \mathrm{D}$ is effective on the release of $\mathrm{Nrg} 4$, and lower circulating $25(\mathrm{OH}) \mathrm{D}$ may, at least in part, mediate the association of plasma Nrg4 with the development of DPN. However, further welldesigned prospective and larger interventional studies are required to confirm our findings and to better understand the underlying mechanisms.

Numerous studies have shown that vasculopathy may play an important role in the pathogenesis of DPN [17]. As is well known, diabetic vascular complications may have a unifying pathogenic mechanism associated with increased oxidative stress. Slattery et al. and our laboratory demonstrated that levels of $\mathrm{Nrg} 4$ gene expression were negatively regulated by oxidative stress [12], and circulating Nrg4 were negatively correlated with markers of oxidative stress (8-iso-prostaglandin $F_{2 \alpha}$ and gamma-glutamyltransferase) [3], raising the possibility that circulating $\mathrm{Nrg} 4$ may be protective against diabetic micro- and macrovascular complications because of its antioxidant properties, and altered level of circulating Nrg4 may be associated with the development of DN and DR. Surprisingly, we found no significant differences in the prevalence of DN and DR, and DN-related markers across quartiles of circulating $\mathrm{Nrg} 4$ and circulating $\mathrm{Nrg} 4$ was also not associated with the prevalence of DN and $\mathrm{DR}$, and DN-related markers, suggesting that circulating Nrg4 levels may not involve in the pathogenesis of diabetic microvascular complications other than DPN, and the three diabetic microvascular complications might have other different pathogenetic mechanisms. Our data coincide with a previous report showing that there was no significant difference in serum Nrg4 level between T2DM patients with and without DR [35], but are not consistent with the studies performed by Kralisch et al. and Zahid Kocak et al. [35, 42]. Kralisch et al. found that circulating Nrg4 was significantly lower in patients with end-stage kidney disease on maintenance renal replacement therapy treatment compared to subjects with an eGFR $>50 \mathrm{ml} / \mathrm{min} / 1.73 \mathrm{~m}^{2}$ and independently associated with a preserved renal function and mRNA expression of $\mathrm{Nrg} 4$ is reduced in adipose tissue depots of mice with diabetic kidney disease as compared to nondiabetic control mice [42]. Similarly, Zahid Kocak and collaborators demonstrted that serum Nrg4 significantly reduced in T2DM patients with DN, and serum Nrg4 was negatively associated with microalbuminuria [35]. The difference between our findings and results of previous studies can be partially explained by selection of different populations, differing sample size, and statistical power. $\mathrm{ABI}$, a surrogate marker for atherosclerosis and predictor of future cardiovascular events, has been widely used to establish the diagnosis of lower extremity PAD, another diabetic macrovascular complication [43, 44]. Our finding that circulating Nrg4 was not related to ABI values and the prevalence of PAD was inconsistent with the results of previous similar studies [13-15]. Jiang et al. found that circulating Nrg4 was inversely associated with subclinical CVD, as evidence by increased carotid intima-media thickness and atherosclerotic plaque in obese adults [13]. A negative correlation between circulating Nrg4 and the presence and severity of coronary artery disease was also reported in another study [14]. Recently, it has been demonstrated that administration of Nrg4 could mitigate the fraction of myocardial infarcts in a mouse model of experimental myocardial ischemia [15]. The discrepant findings between our study and those of previous studies may be partly explained by differing study designs, sample size, selection of patients, duration of diabetes, and medication use. Thus, more evidence is required from longitudinal and randomized controlled studies to validate our findings.

Our study has several limitations that must be acknowledged. Firstly, our results must be carefully interpreted because the cross-sectional study design with small sample size makes it difficult to make any causal inference. Secondly, the present study population consisted of Chinese Han nT2DM patients at a single center, and thus our findings do not apply to other types of diabetes with different ethnicity. Thirdly, 25(OH)D levels affected by many factors, such as dietary supplements, sun exposure, time outdoors, sun-protective behaviors such as use of sunscreen or sun hat, physical activity, season changes, geographic location, sedentary lifestyle and other lifestyle. However, we did not account for these factors because these information is not currently available in our study. Despite this, our study has several advantages. First of all, the DPN was defined using neurological symptoms, reflexes examination, and QST, which were widely used to evaluate whether different types of nerve fibers had neurological dysfunction in clinical screening studies. Secondly, we tentatively explored the 
relationship between circulating $\mathrm{Nrg} 4$ and other diabetic vascular complications, and for the first time found that circulating Nrg4 levels were not associated with the presence of DN, DR and PAD. Last but most importantly, our study is, to our knowledge, the first to evaluate the association between circulating Nrg4 and 25(OH)D in Chinese nT2DM patients, and found that plasma Nrg4 was positively and independently associated with $25(\mathrm{OH}) \mathrm{D}$.

\section{Conclusions}

The present study showed that circulating Nrg4 level significantly decreased in nT2DM patients with DPN, and was independently and negatively correlated with the risk of DPN development. Moreover, circulating Nrg4 levels were independently associated with $25(\mathrm{OH}) \mathrm{D}$, but showed no associations with other diabetic microvascular complications. These findings suggest that decreased circulating Nrg4 may trigger the development of DPN through its close interaction with $25(\mathrm{OH}) \mathrm{D}$ not with other diabetic vascular complications. However, our findings need to be confirmed by more well-designed prospective studies.

\section{Abbreviations \\ ANOVA: One-way analysis of variance; ABI: Ankle-brachial index; BMI: Body mass index; BAT: Brown adipose tissue; Cl: Confidence intervals; CVD: Cardio- vascular disease; CKD-EPI: Chronic kidney disease epidemiology collabora- tion; DPN: Diabetic peripheral neuropathy; DBP: Diastolic blood pressure; DN: Diabetic nephropathy; DR: Diabetic retinopathy; DKD: Diabetic kidney disease; eGFR: Estimated glomerular filtration rate; EGF: Epidermal growth factor; ESKD: End-stage kidney disease; FBG: Fasting blood glucose; Fins: Fasting insulin; GDNF: Glial cell line-derived neurotrophic factor; HDL-C: High-density lipo- protein cholesterol; HbA1c: Glycated hemoglobin A1c; 25(OH)D: 25-hydroxy vitamin D; HOMA-IR: Homeostasis model assessment of insulin resistance; LDL-C: Low-density lipoprotein cholesterol; MSN: Medium spiny neurons; Nrg4: Neuregulin 4; nT2DM: Newly diagnosed type 2 diabetes mellitus; NGF: Nerve growth factor; OGTT: Oral glucose tolerance test; PAD: Peripheral arterial disease; PBG: Postprandial 2 h blood glucose; Q: Quartile; QST: Quantitative sensory testing; RRT: Renal replacement therapy; SD: Standard deviation; SBP: Systolic blood pressure; Scr: Serum creatinine; TC: Total cholesterol; TG: Triglyceride; UACR: Urine albumin-to-creatinine ratio; VPT: Vibration perception threshold. \\ Acknowledgements \\ The authors would like to thank all the colleagues in clinical laboratory center and endocrine laboratory, and all the nurses in our department for their hard work and valuable assistance with this study.}

\section{Authors' contributions}

All the authors contributed significantly to the manuscript. PJY conducted the population study, analyzed and interpreted the data, and drafted the manuscript. ZHZ significantly revised the draft, interpreted the data, and involved in data analyses. YM and YX conducted the study, collected the information and participated in data interpretation. JHZ involved in the sample test, data management and draft revision. QW is the PI of project, who designed the study and critically revised the manuscript. All authors read and approved the final manuscript.

\section{Funding}

This work was supported by the Grants 2016YFC0901200, 2016YFC0901205 from the Ministry Science and Technology of China, Research Grants from Health and Family Planning Commission of Sichuan Province (16129).
Availability of data and materials

The data is available upon reasonable request to the corresponding author.

\section{Ethics approval and consent to participate}

The study was approved by the ethics committee of the Affiliated Hospital of Southwest Medical University. Written informed consent was obtained before the data collection and analysis.

\section{Consent for publication}

Not applicable.

\section{Competing interests}

The authors declare that they have no competing interests.

\section{Author details}

${ }^{1}$ Department of Endocrinology, The Affiliated Hospital of Southwest Medical University, Luzhou, Sichuan 646000, China. ${ }^{2}$ Department of General Medicine, The Affiliated Hospital of Southwest Medical University, Luzhou, Sichuan 646000 , China.

Received: 1 January 2020 Accepted: 9 May 2020

Published online: 19 May 2020

\section{References}

1. Boulton AJ, Malik RA, Arezzo JC, Sosenko JM. Diabetic somatic neuropathies. Diab Care. 2004;27(6):1458-86.

2. Xu F, Zhao LH, Su JB, Chen T, Wang XQ, Chen JF, et al. The relationship between glycemic variability and diabetic peripheral neuropathy in type 2 diabetes with well-controlled HbA1c. Diabetol Metab Syndr. 2014;6(1):139.

3. Yan P, Xu Y, Zhang Z, Gao C, Zhu J, Li H, et al. Decreased plasma neuregulin 4 levels are associated with peripheral neuropathy in Chinese patients with newly diagnosed type 2 diabetes: a cross-sectional study. Cytokine. 2019;113:356-64

4. Zheng Y, Ley SH, Hu FB. Global aetiology and epidemiology of type 2 diabetes mellitus and its complications. Nat Rev Endocrinol. 2018;14(2):88-98.

5. Pfeifer A. NRG4: an endocrine link between brown adipose tissue and liver. Cell Metab. 2015;21(1):13-4.

6. Wang GX, Zhao XY, Meng ZX, Kern M, Dietrich A, Chen Z, et al. The brown fat-enriched secreted factor Nrg4 preserves metabolic homeostasis through attenuation of hepatic lipogenesis. Nat Med. 2014;20(12):1436-43.

7. Zhang P, Kuang H, He Y, Idiga SO, Li S, Chen Z, et al. NRG1-FC improves metabolic health via dual hepatic and central action. JCl Insight. 2018;3(5):e98522.

8. Cai C, Lin M, Xu Y, Li X, Yang S, Zhang H. Association of circulating neuregulin 4 with metabolic syndrome in obese adults: a cross-sectional study. BMC Med. 2016:14(1):165.

9. Dai YN, Zhu JZ, Fang ZY, Zhao DJ, Wan XY, Zhu HT, et al. A case-control study: Association between serum neuregulin 4 level and non-alcoholic fatty liver disease. Metabolism. 2015;64(12):1667-73.

10. Pellegrinelli V, Peirce VJ, Howard L, Virtue S, Türei D, Senzacqua M, et al. Adipocyte-secreted BMP8b mediates adrenergic-induced remodeling of the neuro-vascular network in adipose tissue. Nat Commun. 2018;9(1):4974

11. McElroy SJ, Castle SL, Bernard JK, Almohazey D, Hunter CJ, Bell BA, et al. The ErbB4 ligand neuregulin-4 protects against experimental necrotizing enterocolitis. Am J Pathol. 2014;184(10):2768-78.

12. Slattery ML, Pellatt DF, Mullany LE, Wolff RK. Differential gene expression in colon tissue associated with diet, lifestyle, and related oxidative stress. PLoS ONE. 2015;10(7):e0134406.

13. Jiang J, Lin M, Xu Y, Shao J, Li X, Zhang H, et al. Circulating Neuregulin 4 levels are inversely associated with subclinical cardiovascular disease in obese adults. Sci Rep. 2016;6:36710.

14. Tian QP, Liu ML, Tang CS, Xue L, Pang YZ, Qi YF. Association of circulating neuregulin-4 with presence and severity of coronary artery disease. Int Heart J. 2019;60(1):45-9. 
15. Liu SQ, Tefft BJ, Roberts DT, Zhang LQ, Ren Y, Li YC, et al. Cardioprotective proteins upregulated in the liver in response to experimental myocardial ischemia. Am J Physiol Heart Circ Physiol. 2012;303(12):H1446-58.

16. Lee KO, Nam JS, Ahn CW, Hong JM, Kim SM, Sunwoo IN, et al. Insulin resistance is independently associated with peripheral and autonomic neuropathy in Korean type 2 diabetic patients. Acta Diabetol. 2012;49(2):97-103.

17. Tesfaye S, Chaturvedi N, Eaton SE, Ward JD, Manes C, lonescu-Tirgoviste $C$, et al. Vascular risk factors and diabetic neuropathy. N Engl J Med. 2005;352(4):341-50.

18. Liu Y, Ford BD, Mann MA, Fischbach GD. Neuregulin-1 increases the proliferation of neuronal progenitors from embryonic neural stemcells. Dev Biol. 2005;283(2):437-45.

19. Paramo B, Wyatt $S$, Davies AM. An essential role for neuregulin-4 in the growth and elaboration of developing neocorticalpyramidal dendrites. Exp Neurol. 2018;302:85-92.

20. Rosell M, Kaforou M, Frontini A, Okolo A, Chan YW, Nikolopoulou E, et al. Brown and white adipose tissues: intrinsic differences in gene expression and response to cold exposure in mice. Am J Physiol Endocrinol Metab. 2014:306(8):E945-64.

21. Hayes NV, Newsam RJ, Baines AJ, Gullick WJ. Characterization of the cell membrane-associated products of the Neuregulin 4 gene. Oncogene. 2008;27(5):715-20.

22. Abbas MA. Physiological functions of Vitamin D in adipose tissue. J Steroid Biochem Mol Biol. 2017;165(Pt B):369-81.

23. de Souza WN, Norde MM, Oki É, Rogero MM, Marchioni DM, Fisberg RM, et al. Association between 25-hydroxyvitamin D and inflammatory biomarker levels in a cross-sectional population-based study, São Paulo. Brazil Nutr Res. 2016;36(1):1-8.

24. Walker GE, Ricotti R, Roccio M, Moia S, Bellone S, Prodam F, Bona G. Pediatric obesity and vitamin D deficiency: a proteomic approach identifies multimeric adiponectin as a key link between these conditions. PLoS ONE. 2014;9(1):e83685.

25. Alamdari A, Mozafari R, Tafakhori A, Faghihi-Kashani S, Hafezi-Nejad $N$, Sheikhbahaei $S$, et al. An inverse association between serum vitamin D levels with the presence and severity of impaired nerve conduction velocity and large fiber peripheral neuropathy in diabetic subjects. Neurol Sci. 2015;36:1121-6.

26. Zubair M, Malik A, Meerza D, Ahmad J. 25-Hydroxyvitamin D [25(OH)D] levels and diabetic foot ulcer: is there any relationship? Diab Metab Syndr. 2013;7:148-53.

27. Khan RJ, Gebreab SY, Riestra P, Sims M, Gaye A, Xu R, et al. Associations between vitamin $D$ and cardiovascular disease risk factors in african americans are partly explained by circulating adipokines and C-reactive protein: the jackson heart study. J Nutr. 2016;146(12):2537-43.

28. Jung CH, Kim KJ, Kim BY, Kim CH, Kang SK, Mok JO. Relationship between vitamin D status and vascular complications in patients with type 2 diabetes mellitus. Nutr Res. 2016;36(2):117-24.

29. Groves NJ, McGrath JJ, Burne TH. Vitamin D as a neurosteroid affecting the developing and adult brain. Annu Rev Nutr. 2014;34:117-41.

30. Dikker O, Bekpinar S, Ozdemirler G, Uysal M, Vardar M, Atar S, et al. Evaluation of the relation between omentin-1 and vitamin $D$ in postmenopausal women with or without osteoporosis. Exp Clin Endocrinol Diab. 2018;126(5):316-20.

31. Levey AS, Stevens LA, Schmid CH, Zhang YL, Castro AF 3rd, Feldman HI, et al. A new equation to estimate glomerular filtration rate. Ann Intern Med. 2009:150(9):604-12

32. Yan $P$, Zhang Z, Wan Q, Zhu J, Li H, Gao C, et al. Association of serum uric acid with bone mineral density and clinical fracturesin Chinese type 2 diabetes mellitus patients: a cross-sectional study. Clin Chim Acta. 2018;486:76-85

33. Paramo B, Wyatt $S$, Davies AM. Neuregulin-4 is required for the growth and elaboration of striatal medium spiny neuron dendrites. J Neuropathol Exp Neurol. 2019. https://doi.org/10.1093/jnen/nlz046.

34. Zhang L, Fu Y, Zhou N, Cheng X, Chen C. Circulating neuregulin 4 concentrations in patients with newly diagnosed type 2 diabetes: a crosssectiona study. Endocrine. 2017:57(3):535-8.

35. Kocak MZ, Aktas G, Atak BM, Duman TT, Yis OM, Erkus E, et al. Is Neuregulin- 4 a predictive marker of microvascular complications in type 2 diabetes mellitus? Eur J Clin Invest. 2020:50(3):e13206.

36. Shehab D, Al-Jarallah K, Mojiminiyi OA, Al Mohamedy H, Abdella NA. Does Vitamin D deficiency play a role in peripheral neuropathy in Type 2 diabetes? Diab Med. 2012;29(1):43-9.

37. Uro M, Beauchet O, Cherif M, Graffe A, Milea D, Annweiler C. Age-related vitamin $D$ deficiency is associated with reduced macular ganglion cell complex: a cross-sectional high-definition optical coherence tomography study. PloS ONE. 2015;10(6):e0130879.

38. Maser RE, Lenhard MJ, Pohlig RT. Vitamin D insufficiency is associated with reduced parasympathetic nerve fiber function in type 2 diabetes. Endocr Pract. 2015;21(2):174-81.

39. Gupta GK, Agrawal T, DelCore MG, Mohiuddin SM, Agrawal DK. Vitamin D deficiency induces cardiac hypertrophy and inflammation in epicardial adipose tissue in hypercholesterolemic swine. Exp Mol Pathol. 2012;93(1):82-90

40. Lorente-Cebrián S, Eriksson A, Dunlop T, Mejhert N, Dahlman I, Aström G, et al. Differential effects of 1a, 25-dihydroxycholecalciferol on MCP-1 and adiponectin production in human white adipocytes. Eur J Nutr. 2012;51(3):335-42.

41. Chen Z, Wang GX, Ma SL, Jung DY, Ha H, Altamimi T, et al. Nrg4 promotes fuel oxidation and a healthy adipokine profile to ameliorate diet-induced metabolic disorders. Mol Metab. 2017;6(8):863-72.

42. Kralisch S, Hoffmann A, Klöting N, Frille A, Kuhn H, Nowicki M, et al. The brown fat-secreted adipokine neuregulin 4 is decreased in human and murine chronic kidney disease. Eur J Endocrinol. 2019;181(2):151-9.

43. Lau JF, Weinberg MD, Olin JW. Peripheral artery disease Part 1: clinical evaluation and noninvasive diagnosis. Nat Rev Cardiol. 2011;8(7):405-18.

44. Marso SP, Hiatt WR. Peripheral arterial disease in patients with diabetes. J Am Coll Cardiol. 2006:47(5):921-9.

\section{Publisher's Note}

Springer Nature remains neutral with regard to jurisdictional claims in published maps and institutional affiliations.

Ready to submit your research? Choose BMC and benefit from

- fast, convenient online submission

- thorough peer review by experienced researchers in your field

- rapid publication on acceptance

- support for research data, including large and complex data types

- gold Open Access which fosters wider collaboration and increased citations

- maximum visibility for your research: over $100 \mathrm{M}$ website views per year

At $\mathrm{BMC}$, research is always in progress.

Learn more biomedcentral.com/submissions 